Original Article (short paper)

\title{
Does the number of sets in a resistance exercise session affect the fast and slow phases of post-exercise cardiac autonomic recovery?
}

\author{
Carlos Janssen Gomes da Cruz ${ }^{1,2}$ (1) , Luiz Guilherme Grossi Porto ${ }^{2}$ (D) , Deleon de Souza Pires ${ }^{1}$ (D) , Rivadávio Fer- \\ nandes Batista de Amorim ${ }^{3}$ (i) , Frederico Santos de Santana ${ }^{1}$ (D) , Guilherme Eckhardt Molina ${ }^{2}$ (i) \\ ${ }^{1}$ Centro Universitário Euro Americano, Grupo de Estudos e Pesquisas em Função Autônoma Cardíaca, Brasí- \\ lia, DF, Brasil, ${ }^{2}$ Universidade de Brasília, Faculdade de Educação Física, Laboratório de Fisiologia do Exer- \\ cício, Brasília, DF, Brasil; ${ }^{3}$ Universidade de Brasília, Faculdade de Medicina, Brasília, DF, Brasil
}

\begin{abstract}
Aims: The purpose of this study was to evaluate the acute effects of different resistance exercise (RE) volumes on postexercise cardiac autonomic modulation in men. Methods: Ten young men $(25.5 \pm 4.9$ years, 24.8 $\left.\pm 2.1 \mathrm{~kg} / \mathrm{m}^{2}\right)$ performed 3 trials of RE with 1,2 or 3 sets (48-72 h between each trial) of $10-12$ repetitions (70\% of the one-maximum repetition) of bench press, leg press, and barbell row. Heart rate variability (HRV) was assessed at the $1^{\text {st }}$ and $5^{\text {th }}$ minutes of recovery (fast phase) and 3 consecutive 5 -minute intervals from the $5^{\text {th }}$ to $20^{\text {th }}$ minute of recovery (slow phase). Parasympathetic and global modulations were assessed using the SD1 and SD2 indices of HRV, respectively. The comparison of the interventions was performed using the Friedman and Wilcoxon tests $(p \leq 0.05)$. Results: Lower parasympathetic modulation was identified after 2 and 3 sets compared to 1 set in both the fast and slow recovery phases $(\mathrm{p}=0.004-0.05)$. Lower global modulation was identified after 3 sets compared to 1 set in both fast and slow recovery phases $(\mathrm{p}=0.005-0.01)$. No differences in post-exercise parasympathetic and global modulation were observed between 2 and 3 sets. Conclusion: We concluded that 2 and 3 sets of RE compared to 1 set promoted higher autonomic reduction on the post-exercise phase, which should be considered by coaches when prescribing an RE program for untrained participants or intend to manipulate the postexercise organic recovery.
\end{abstract}

Keywords: strength training, heart rate variability, vagal reactivation, heart rate recovery, autonomic nervous system

\section{Introduction}

During exercise, an increase in cardiac sympathetic activity and a decrease in cardiac parasympathetic modulation occurs to increase blood pressure and cardiac output according to the peripheral metabolic demand ${ }^{1}$. Conversely, the postexercise recovery period is characterized by sympathetic withdrawal with simultaneous cardiac parasympathetic reactivation (PR), which returns the heart rate (HR) and blood pressure to resting levels ${ }^{2}$. In the exercise field, postexercise PR has been used to identify acute and chronic adjustments/adaptations in the cardiac autonomic function that are related to different modalities of exercise training ${ }^{3-5}$.

In this scenario, the use of heart rate variability (HRV), a proxy measure of cardiac autonomic modulation, to monitoring PR has gained attention in the scientific literature as a low cost and non-invasive alternative to manage the exercise-induced internal overload ${ }^{6,7}$. Specifically, it has been proposed that the adjustment of the training load according to cardiac autonomic recovery (AR) allows to manage the balance between stimulus and recovery, can contribute to increased physical performance, and reducing the risk of nonfunctional overreaching ${ }^{9}$. Thus, a better knowledge concerning the acute effects of different resistance exercise (RE) training programs on cardiac autonomic modulation capacity can improve exercise protocols that take into account the cardiac autonomic status, contributing to minimize the risk of exercise-induced negative response.
Although previous studies report a negative association between aerobic exercise volume/intensity and cardiac $\mathrm{PR}^{10-15}$, little scientific evidence about the acute effects of different RE program protocols on PR has been published ${ }^{16-19}$. In this scenario, a significant contribution was provided by Figueiredo et al. ${ }^{19}$, demonstrating a dose-dependent suppressive effect of $\mathrm{RE}$ volume on postexercise cardiac autonomic modulation. In the aforementioned study, experienced resistance training participants underwent 8 different RE performing 1, 3, and 5 sets per exercise. Thus, despite relevant contributions provided by Figueiredo et al. ${ }^{19}$, it is necessary to investigate the autonomic impact of the different number of sets and exercises in a RE session performed by untrained or inexperienced participants on resistance training.

Because of the functional and practical relevance and the relation to exercise prescription and recovery plans, it is crucial to better understand how different RE volumes can influence cardiac parasympathetic and global modulation throughout the post-exercise recovery phase. Thus, the present study aimed to investigate the acute effects of different $R E$ volumes $(1,2$, or 3 sets per exercise) on the fast and slow phases of postexercise parasympathetic and global reactivation, assessed by HRV, in a sample of untrained men. In addition, the study evaluated the correlation between RE volume and different markers of postexercise cardiac autonomic modulation. 


\section{Methods}

\section{Study Design, Sample and Ethical Issues}

This quasi-experimental study was conducted with a sample composed of 10 young men $(25.5 \pm 4.9$ years, $24.8 \pm 2.1 \mathrm{~kg} /$ $\mathrm{m}^{2}$ ) with no or little experience in RE programs ( $<6$ months). The following inclusion criteria were used: i) men with RE experience between 0 and 6 months, ii) no involvement in exercise training programs at least 6 months before the start of resistance training, iii) without any self-reported chronic diseases, iv) nonsmokers and v) no previous experience in sports competitions. The volunteers were advised to abstain from stimulants, alcoholic beverages, and physical activity for at least $48 \mathrm{~h}$ prior to the test and to have their last meal 2-3 $\mathrm{h}$ before the intervention. Subjects who did not follow the aforementioned criteria or who started drug treatment during the data collection period were excluded ( 2 participants).

All participants were informed about the risks and benefits of the investigation before beginning the test, and all signed informed consent to participate. The study protocol was approved by the Institutional Ethical Committee on Human Research (protocol number: 306.359) in compliance with the National Research Ethics System Guidelines and the Declaration of Helsinki.

\section{Experimental protocol}

All participants underwent a total of six visits to the laboratory between 2:00 and 5:00 p.m. In the first visit, anthropometric variables (body mass and height) were measured, and participants were submitted to a familiarization procedure with the RE adopted in this study. In the second visit, maximum strength (one repetition maximum - 1RM) on the bench press (free weight), leg press $\left(45^{\circ}\right)$, and barbell row (free weight) were measured. In the subsequent visit, the $1 \mathrm{RM}$ retest was performed, as detailed below. Afterward, the sample population randomly underwent 3 RE protocols, consisting of 1,2 , or 3 sets per exercise with an interval of 48-72 $\mathrm{h}$ between each trial.

\section{One repetition maximum (1RM) test}

The 1RM tests were performed by two experienced investigators on nonconsecutive days (48-72 $\mathrm{h}$ of the interval) according to previous recommendations ${ }^{20}$. Initially, sets of 8 , 3 , and 1 repetition were executed with loads corresponding to 30,75 , and $100 \%$ of the predicted $1 \mathrm{RM}$ (according to the previous experience of the participants) in each exercise. Subsequently, load increments of 5\% were added for each attempt to perform one complete repetition, with 5-minute inter-set resting intervals, until concentric failure was reached. At failure, an additional attempt with a weight approximately midway between the last successful and the last failed lift was executed. The maximal amount of weight lifted in one complete repetition in each exercise was considered the 1RM load.

The $1 \mathrm{RM}$ retest was performed $48-72 \mathrm{~h}$ after the first assessment to confirm the maximum strength for each exercise. If a difference $>5 \%$ was observed between the first and second measures, a third 1RM test was performed, and the higher load was used for the exercise prescription $(n=1)$. Finally, if the 1RM loads were not reached after 5 attempts, a new test was performed after $48-72 \mathrm{~h}$ to minimize fatigue effects and increase the accuracy of the $1 R M(n=2)$.

\section{Resistance exercise sessions}

The RE sessions consisted of the execution of 1, 2 or 3 sets of 10-12 repetitions on the bench press (free weight), leg press $\left(45^{\circ}\right)$ and barbell row (free weight) with a load corresponding to $70 \%$ of the 1RM (1-minute rest interval between sets). The volume and intensity of training sessions were adopted in accordance with the recommendations of the American College of Sports Medicine to improve muscular strength and hypertrophy in novice individuals ${ }^{21}$ when appropriately evaluated and advised by a health professional.

\section{Resting hemodynamic and respiratory variables}

After 10 minutes in the supine position, HR, systolic blood pressure (SBP) and diastolic blood pressure (DBP) were assessed. The HR and blood pressure were assessed using a v800 Polar $\AA$ HR monitor ${ }^{22}$ and the auscultatory method (Premium ${ }^{\circledR}$, Brazil), respectively. Two measurements of blood pressure were performed in the right arm of participants, and the average of measurements was calculated. If the difference between measurements was greater than $5 \mathrm{mmHg}$, a new measurement was performed, and the two measurements with a difference $<5 \mathrm{mmHg}$ were considered for analysis. In addition, the subjects remained to breathe spontaneously and had their respiratory rate (RR) visually monitored and counted from the number of chest expansion and retraction for 1 minute. All physiological and functional measurements were performed by the same researcher in all participants of this study.

\section{Resting and post-exercise cardiac autonomic evaluation}

After 15 minutes of resting in the supine position, a five-minute $\mathrm{iRR}$ segment was recorded to obtain a resting autonomic characterization of the participants before each exercise session. After the last set was performed in each RE trial, the participants were instructed to adopt the supine position on a stretcher within $60 \mathrm{~s}$. The fast and slow postexercise phases of cardiac $\mathrm{AR}^{2}$ were evaluated by HRV analysis in the supine position. The fast component of AR was assessed at the $1^{\text {st }}\left(\mathrm{AR}_{1 \min }\right)$ and $5 \mathrm{t}^{\mathrm{h}}\left(\mathrm{AR}_{5 \min }\right)$ minutes of the post-exercise 
HRV analysis, and the slow phase was assessed at 3 consecutive 5-minute R-R intervals (iRR) segments obtained from the 5 th to 20 th minute of passive recovery $\left(A R_{10 \min }, A R_{15 \text { min, }}\right.$ and $\mathrm{AR}_{20 \mathrm{~min}}$ ).

The time-series of iRR segments was obtained using a v800 Polar ${ }^{\circledR}$ cardiac monitor ${ }^{22,23}$. After data collection, the iRR segments were visually inspected and analyzed using Kubios HRV software, version 2.024. The software computes all the commonly used time-domain and frequency-domain HRV parameters and several nonlinear parameters. There are several adjustable analysis settings through which the analysis methods can be optimized for different data. The analysis results can be saved as an ASCII text file (easy to import into MS Excel or SPSS). Eventual artifacts were removed without adding a new iRR to the temporal series. However, when artifacts reached more than $1 \%$ of the total iRR segment, the participant's data were excluded from the analysis $(n=1)^{25}$.

Following artifact adjustments, the standard deviation of the instantaneous beat-to-beat variability (SD1) and the standard deviation of the long-term continuous iRR (SD2) of the Poincaré scatterplot map was used to assess parasympathetic and global (sympathetic + parasympathetic) modulation, respectively ${ }^{26}$. The Poincaré method allows for a valid ${ }^{26}$ and reliable ${ }^{27}$ measurement of cardiac autonomic modulation during nonstationary conditions (i.e., during and after exercise training and testing) and was used to assess the cardiac autonomic modulation at resting supine position (SD1 $1_{\text {resting }}$ and $\mathrm{SD} 2_{\text {resting }}$ ) and in the fast $\left(\mathrm{SD} 1_{1 \mathrm{~min}}, \mathrm{SD} 2_{1 \mathrm{~min}}\right.$, $\mathrm{SD} 1_{5 \min ,}$ and $\mathrm{SD} 2_{5 \min }$ ) and slow $\left(\mathrm{SD} 1_{10 \mathrm{~min}}, \mathrm{SD} 2_{10 \mathrm{~min}}, \mathrm{SD} 1_{15 \mathrm{~min}}\right.$, $\left.\mathrm{SD} 2{ }_{15 \mathrm{~min}}, \mathrm{SD} 1_{20 \mathrm{~min}}, \mathrm{SD} 2_{20 \mathrm{~min}}\right)$ postexercise recovery phases.

\section{Statistical analyses}

The normality hypothesis was rejected by the ShapiroWilk test for some variables. Thus, a nonparametric approach was adopted for descriptive and inferential statistics. Data are shown as medians and interquartile ranges, and Friedman or Wilcoxon tests were used when appropriate. Additionally, Spearman's correlation coefficient was used to evaluate the correlation between the total RE volume of each training session and postexercise cardiac autonomic modulation. To verify the relative reliability between the two valid 1RM strength tests, intraclass correlation coefficients (ICC) and their respective confidence intervals (95\% CIs) were calculated.

Differences and correlations were considered statistically significant when the type I error probabilities were less than or equal to $5 \%(\mathrm{p} \leq 0.05)$. Whenever differences were identified by a Bonferroni post hoc test, the effect size (ES) was calculated by the formula: $E S=Z / \sqrt{ } N$. Since the effect size was calculated from paired data, its analysis was restricted to the differences observed to avoid excessive data presentation (i.e., 1 vs. 2 sets, 1 vs. 3 sets and 2 vs. 3 sets for 18 dependent variables). Considering an alpha error of 0.05 and a moderate ES (0.5), the statistical power of this study was 0.56 . The statistical power calculation and statistical analysis were performed using GPower software (v.3.7.1) and the Statistical Package for the Social Sciences (SPSS-v.21), respectively. Figure composition was performed using Graphpad Prism (v.7).

\section{Results}

The ICC values revealed high reliability between the 1RM strength test for the bench press $(0.96$, CI 95\% $=0.86-0.99)$, leg press $\left(45^{\circ}\right)(0.98$, CI $95 \%=0.94-0.99)$ and barbell row $(0.94$, CI $95 \%=0.80-0.98)$.

No differences were observed between the pre-exercise resting HR $(p=0.10), \operatorname{SBP}(p=0.64), \operatorname{DBP}(p=0.36)$ and RR ( $p=0.96)$ for the 3 RE trials (Table 1). Similarly, no differences were observed for the pre-exercise SD1 $(p=0.91)$ or SD2 $(p=0.67)$ indices for the different protocols (All exercise and postexercise HRV analysis are shown in Figure 1).

Compared to the resting parasympathetic measures, all RE protocols were characterized by low values of $S D 1_{1 \text { min }}(p=0.005$ $0.01 ; \mathrm{ES}=0.78-0.88), \mathrm{SD} 1_{5 \min }(\mathrm{p}=0.004-0.005, \mathrm{ES}=0.87-0.88)$, $\mathrm{SD} 1_{10 \min }(\mathrm{p}=0.005-0.02, \mathrm{ES}=0.72-0.88), \mathrm{SD} 1_{15 \min }(\mathrm{p}=0.005-0.03$, $\mathrm{ES}=0.63-0.88)$ and $\mathrm{SD}_{20 \mathrm{~min}}(\mathrm{p}=0.007-0.01, \mathrm{ES}=0.75-0.85)$ throughout the recovery phase.

Although low values of SD2 were observed for all RE protocols during the fast recovery phase compared to the resting measures $\left[\mathrm{SD} 2_{1 \min }(\mathrm{p}=0.004-0.005, \mathrm{ES}=0.87-0.88)\right.$, $\left.\mathrm{SD} 2_{5 \min }(\mathrm{p}=0.004-0.005, \mathrm{ES}=0.87-0.88)\right]$, only the 2 - and 3 -set protocols resulted in lower values of SD2 ${ }_{10 \min }$ (1 set: $\mathrm{p}=0.33, \mathrm{ES}=0.31 ; 2$ sets: $\mathrm{p}=0.005, \mathrm{ES}=0.88 ; 3$ sets: $\mathrm{p}=0.005, \mathrm{ES}=0.88), \mathrm{SD} 2_{15 \min }(1 \mathrm{set}: \mathrm{p}=0.50, \mathrm{ES}=0.20 ; 2$ sets: $p=0.005, E S=0.88 ; 3$ sets: $p=0.005, E S=0.88)$ and $\mathrm{SD}_{20 \text { min }}(1$ set: $\mathrm{p}=0.24, \mathrm{ES}=0.37 ; 2$ sets: $\mathrm{p}=0.005, \mathrm{ES}=0.88$; 3 sets: $p=0.005, E S=0.88$ ) during the slow recovery phase.

Comparing the fast recovery phase of PR between the RE trials, lower values of SD1 were identified after 2 $\left(\mathrm{SD}_{1 \text { min }}: \mathrm{p}=0.002, \mathrm{ES}=1.0 ; \mathrm{SD}_{5 \min }: \mathrm{p}=0.01, \mathrm{ES}=0.91\right)$ and 3 sets $\left(S D 1_{1 \min }: p=0.02, E S=0.84\right.$ SD $_{5 \min }: p=0.004$, $\mathrm{ES}=0.91)$ compared to 1 set. Additionally, lower values of $\mathrm{SD} 2$ were identified after 3 sets compared to 1 set $\left(\mathrm{SD} 2_{1 \mathrm{~min}}\right.$ : $\left.\mathrm{p}=0.005, \mathrm{ES}=0.98 \mathrm{SD}_{5 \min }: \mathrm{p}=0.05, \mathrm{ES}=0.75\right)$. No differences were observed between 2 and 3 sets for all HRV indices or between 1 and 2 sets for SD2 indices ( $\mathrm{p}>0.05)$.

During the slow recovery phase, lower values of SD1 and SD2 were observed after 3 sets compared to 1 set in the $\mathrm{AR}_{10 \min }\left(\mathrm{SD} 1_{10 \mathrm{~min}}-\mathrm{p}=0.005, \mathrm{ES}=1.0 ; \mathrm{SD} 2_{10 \mathrm{~min}}-\mathrm{p}=0.01\right.$, $\mathrm{ES}=0.96), \mathrm{AR}_{15 \mathrm{~min}}{ }^{1 \mathrm{mD} 1_{15 \mathrm{~min}}}{ }^{-\mathrm{p}}=0.005, \mathrm{ES}=1.0 ; \mathrm{SD} 2_{15 \mathrm{~min}^{-}}{ }^{-}$ $\mathrm{p}=0.005, \mathrm{ES}=1.0)$ and $\mathrm{AR}_{20 \min }\left(\mathrm{SD} 1_{20}-\mathrm{p}=0.005, \mathrm{ES}=1.0\right.$; $\left.\mathrm{SD} 2_{20 \min }-\mathrm{p}=0.005, \mathrm{ES}=1.0\right)$. No differences were observed between the postexercise values of SD2 between the 1-set vs 2 -set $(p>0.05)$ or between the 2 -set vs 3 -set $(p>0.05)$ protocols in the slow recovery phase.

Last, low to moderate negative correlations were observed between the RE volume and all post-exercise HRV indices $\left(r_{s}=-0.67\right.$ to $-0.43, p<0.001$ to 0.01 ; Figure 2), except for the $\mathrm{SD} 2_{5 \min }\left(\mathrm{r}_{\mathrm{s}}=-0.25, \mathrm{p}=0.18\right)$. 

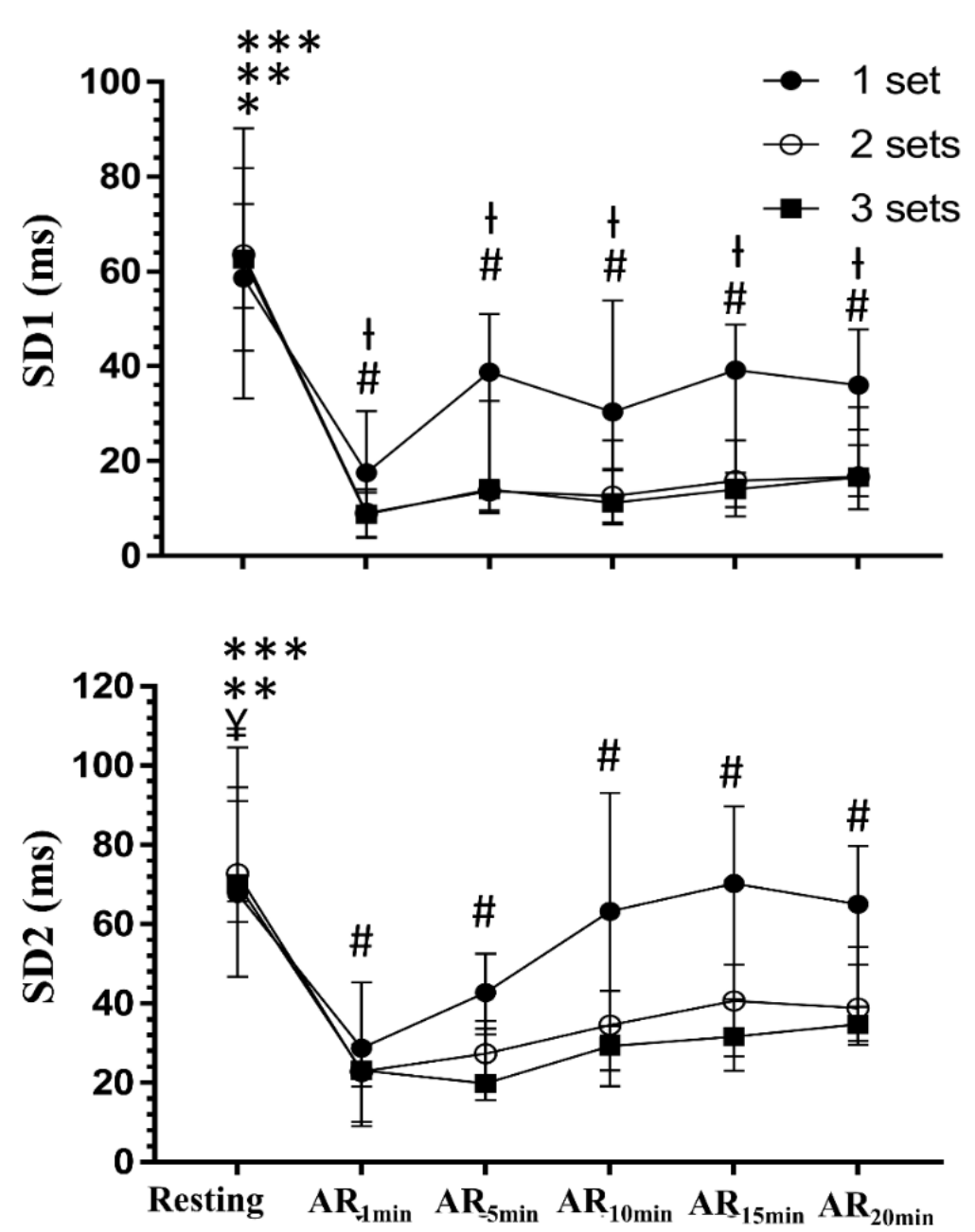

Figure 1- Median and interquartile range of cardiac parasympathetic (SD1) and global (SD2) modulation in resting supine position (Resting) and during 1st (AR1min), 5th (AR5min), 10th (AR10min), 15th (AR15), and 20th (AR20min) minutes of postexercise recovery phases.

\#Significant difference compared to 3 sets (Friedman test, $\mathrm{p}<0.05$ );

I Significant difference compared to 2 sets (Friedman test, $\mathrm{p}<0.05$ );

*Significant difference between resting 1 -set protocol and all intragroup postexercise analysis (Wilcoxon test, $\mathrm{p}<0.05$ );

**Significant difference between resting 2 -set protocol and all intragroup postexercise analysis (Wilcoxon test, $\mathrm{p}<0.05$ );

$* * *$ Significant difference between resting 3 -set protocol and all intragroup postexercise analysis (Wilcoxon test, $\mathrm{p}<0.05$ );

$¥$ Significant difference between resting 1-set protocol and intragroup postexercise AR1min and AR5min $(\mathrm{p}<0.05)$.

Table 1- Median (interquartile range) cardiovascular and respiratory pre-exercise measurements assessed in the three occasions.

\begin{tabular}{|c|c|c|c|c|}
\hline Variables & 1 Set & 2 Sets & 3 Sets & $p$ \\
\hline HR (bpm) & $55(52-65)$ & $57(56-59)$ & $55(54-57)$ & 0.10 \\
\hline SBP (mmHg) & $119(111-124)$ & $119(111-120)$ & $120(110-121)$ & 0.64 \\
\hline DBP (mmHg) & $79(80-81)$ & $73(70-81)$ & $76(70-80)$ & 0.36 \\
\hline RR (Cycles/min) & $15(13-17)$ & $15(13-18)$ & $15(13-16)$ & 0.96 \\
\hline
\end{tabular}

Nonparametric Friedman test. $\mathrm{HR}=$ resting heart rate, $\mathrm{SBP}=$ systolic blood pressure, $\mathrm{DBP}=$ diastolic blood pressure, $\mathrm{RR}=$ respiratory rate. 

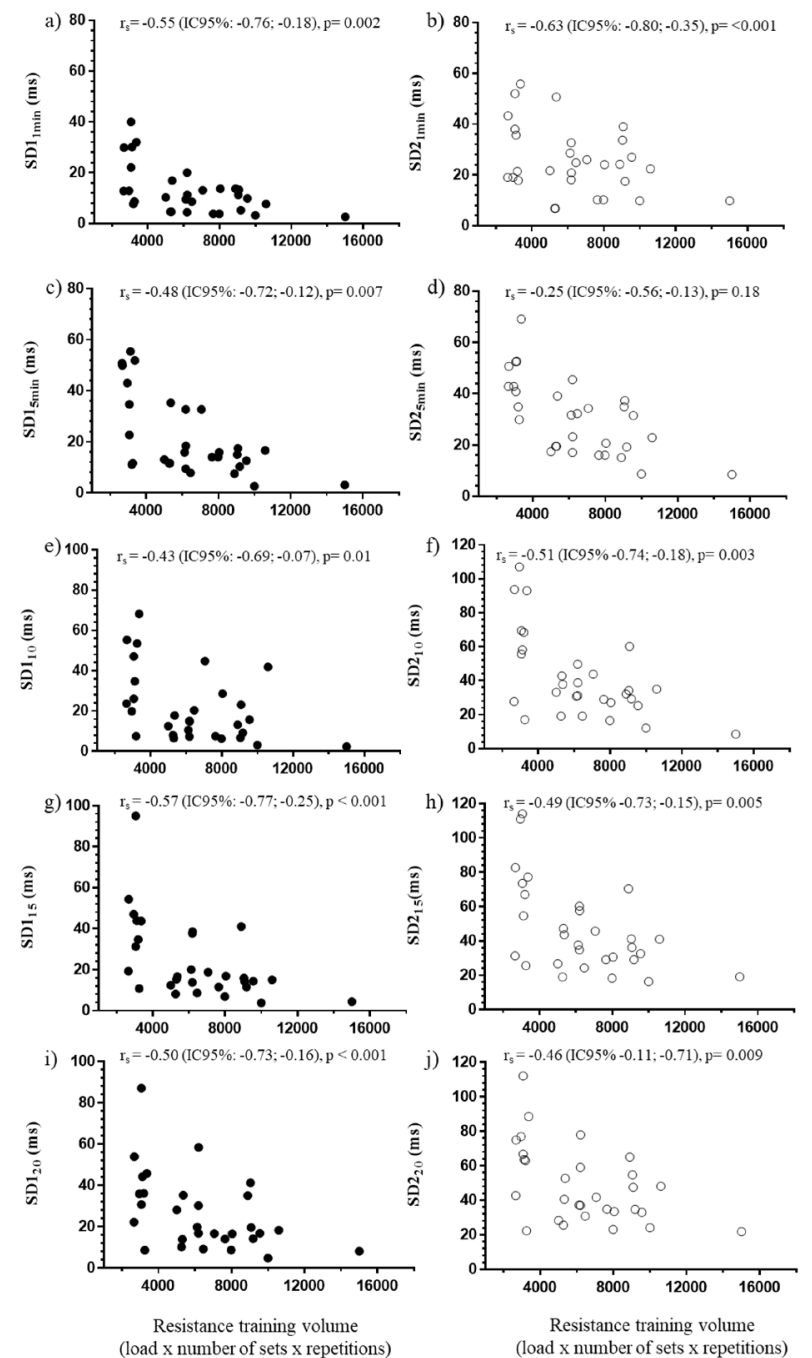

Figure 2- Correlation between resistance exercise volume (load $\mathrm{x}$ number of sets $\mathrm{x}$ number of repetitions) and cardiac parasympathetic (SD1-closed circle) and global (SD2-open circle) modulation during the 1st (a-b), 5th (c-d) 10th (e-f), 15th (g-h), and 20th minutes of the post-exercise recovery phase.

\section{Discussion}

This study demonstrated that, in young men, the magnitude of cardiac parasympathetic and the global reactivation after a RE session are dose-dependent. Specifically, 2 and 3 sets of $\mathrm{RE}$ resulted in lower postexercise PR during the fast and slow recovery phases as compared to 1 set, and 3 sets of RE resulted in lower global reactivation as compared to 1 set during fast and slow postexercise recovery phases. Moreover, a negative relationship was observed between $\mathrm{RE}$ volume and postexercise parasympathetic and global indices.

Corroborating our results, Figueiredo, et al. ${ }^{19}$ observed high parasympathetic reduction after 5 sets of RE as compared to 1 or 3 sets, demonstrating the dose-dependent suppressive effect of RE volume on postexercise autonomic measures. However, the RE protocol adopted in the aforementioned study consisted of $8 \mathrm{RE}$, and participants were experienced in RT (>6 months), while in the present study, inexperienced participants ( $<6$ months) performed only 3 RE for large muscle groups. Thus, our findings demonstrated that even a low volume of RE ( $3 \mathrm{RE}$ ) promotes a dose-dependent (number of sets) parasympathetic and global reduction during the fast and slow AR phases.

The secondary analysis of correlation also supports the strength of the primary outcomes. The negative correlation observed between the RE volume and the postexercise parasympathetic/global modulation shows that the increase in RE volume promotes simultaneous postexercise cardiac parasympathetic and global reduction. These results are in accordance with previous studies of cycling ${ }^{15}$ and running ${ }^{28}$, suggesting that increases in training volume may reduce PR regardless of the exercise modality.

The physiological mechanisms that might explain the results are beyond the purpose of the present investigation, but some potential mechanisms can be considered. During exercise, central and peripheral mechanisms promote sympathetic activation and parasympathetic withdrawal to increase cardiac output and blood pressure according to the metabolic demand ${ }^{1,29}$. On the other hand, in the postexercise 
recovery period, the reduction of central command and muscle afferent activities restores the arterial baroreflex, which results in a rapid PR and HR reduction (fast recovery phase $)^{2}$. Subsequently, parasympathetic modulation remains temporarily depressed mainly due to thermoregulation and the gradual clearance of metabolites and catecholamines released during exercise (slow phase) ${ }^{2}$. Thus, it is plausible to infer that the negative correlation between RE volume and postexercise AR results from greater central and peripheral parasympathetic depression and/or sympathetic activation during high-volume RE. However, all of the aforementioned physiological mechanisms need to be confirmed in future studies.

Taken together, these findings suggested that the highest volume ( 3 sets) reduces the degree of PR as well as the global modulation during the fast and slow recovery phases compared to a single-set RE session. On the other hand, compared to 1 set, 2 sets reduced the fast and slow components of PR without changing the global modulation throughout the recovery phase, which may have been dependent on the sample size. Finally, it is important to note that 20 minutes of passive recovery was not enough to restore the pre-exercise resting autonomic profile even in the single-set session.

The results of this study are clinically and functionally relevant and have important practical implications, mainly for trainers/coaches. Although the acute parasympathetic reduction observed throughout the recovery phases might be a physiological response to exercise training ${ }^{2}$, it is important to consider that a persistent parasympathetic depression is an indication of an imbalance between stimulus and recovery and can result in negative organic adaptations ${ }^{9}$. Given this, our results suggest that the prescription of low-volume RE could be an appropriate strategy to minimize the postexercise cardiac autonomic reduction and its possible negative consequences in participants who present a pre-exercise autonomic depressed profile. Thus, training prescriptions based on cardiac autonomic status has been recommended as a promising tool for fine adjustment of a training pro$\operatorname{gram}^{8,30}$. Therefore, the current findings suggest that exercise professionals should consider the effects of RE volume on cardiac autonomic modulation in addition to the possible neuromuscular chronic effects ${ }^{31,32}$.

The main limitations of the present study are the sample size and the absence of ventilatory, sympathetic, and metabolic analyses during and after exercise. All these variables can influence cardiac autonomic modulation ${ }^{2,33}$ and could contribute to understanding the physiological mechanisms related to the delayed postexercise PR and global modulation observed after 3 sets of RE. However, it is important to clarify that even though the results are based on limited sample size, our findings are consistent among different analyses (comparisons and correlations) and time frames of analysis (1 to 20 minutes). Additionally, differences between trials were accompanied by moderate to large effect sizes. Therefore, in contrast to situations in which studies with small sample sizes do not have enough statistical power to exclude the null hypothesis, the confirmation of the alternative hypothesis, i.e., the observation of significantly reduced PR after a low volume of RE trials in small sample size, reinforces the findings. Finally, the possible impact of muscular soreness on HRV analysis was not investigated in this study, but the similarity between pre-exercise cardiorespiratory variables and HRV measures in different protocols indicate a complete AR between trials.

Therefore, the present study investigated the effect of RE volumes on postexercise AR in healthy young men with little or no experience in $\mathrm{RE}(<6$ months). Future investigations should be performed to investigate the acute and chronic effects of different RE designs on the cardiac autonomic regulation of high-cardiovascular-risk patients. However, it is important to highlight that a) due to the expected cardiac autonomic impairment among individuals with high cardiovascular risk or patients with cardiovascular disease ${ }^{34,35}$, it is plausible to expect similar or worse results in terms of the postexercise cardiac autonomic regulation in those individuals as compared to the participants in the present study; and b) that exercise-induced autonomic and non-autonomic negative cardiovascular events are also present in asymptomatic individuals, although fewer frequently ${ }^{36,37}$. Thus, it is wise to adopt preventive measures to minimize exercise-induced cardiovascular risk, even in low-risk patients, especially untrained patients ${ }^{36}$.

\section{Conclusions}

We concluded that 2 and 3 sets of RE compared to 1 set promote higher autonomic reduction on the postexercise recovery phase, which should be considered by coaches when prescribing an RE program for untrained participants or intend to manipulate the postexercise organic recovery.

\section{References}

1. White DW, Raven PB. Autonomic neural control of heart rate during dynamic exercise: revisited. J Physiol. 2014; 592(12):2491-500.

2. Michael S, Graham KS, Davis GMO. Cardiac Autonomic Responses during Exercise and Post-exercise Recovery Using and Systolic Time Intervals-A Review. Front Physiol. 2017;8:1-19.

3. Michael S, Jay O, Graham KS, Davis GM. Influence of exercise modality on cardiac parasympathetic and sympathetic indices during post-exercise recovery. J Sci Med Sport. 2018;21:1079-84.

4. Trojbicz LR, Damasceno MV, Pasqua LA, Gaspari AF, LimaSilva AE, Bertuzzi R. Parasympathetic activity delayed after self-paced exercise. Eur J Sports Sci. 2018;18:842-50.

5. Nakamura M, Hayashi K, Aizawa K, Mesaki N, Kono I. Effects of regular aerobic exercise on post-exercise vagal reactivation in young female. Eur J Sports Sci. 2013;13:674-80.

6. Singh N, Moneghetti KJ, Christle JW, Hadley D, Froelicher V, Plews D. Heart Rate Variability: An Old Metric with New Meaning in the Era of Using mHealth technologies for Health and 
Exercise Training Guidance. Part Two: Prognosis and Training. Arrhythmia Electrophysiol Rev. 2018;7:247-55.

7. Stanley J, Peake JM, Buchheit M. Cardiac parasympathetic reactivation following exercise: implications for training prescription. Sports Med. 2013;43:1259-77.

8. Javaloyes A, Sarabia JM, Lamberts RP, Moya-Ramon M. Training Prescription Guided by Heart-Rate Variability in Cycling. Int J Sports Physiol Perform. 2019;14:23-32.

9. Kajaia T, Maskhulia L, Chelidze K, Akhalkatsi V, Kakhabrishvili $Z$. The effects of non-functional overreaching and overtraining on the autonomic nervous system function in highly trained athletes. Georgian Med News. 2017;264:97-103.

10. Buchheit M, Laursen PB, Ahmaidi S. Parasympathetic reactivation after repeated sprint exercise. Am J Physiol Heart Circ Physiol. 2007;293:133-41.

11. Gladwell VF, Sandercock GR, Birch SL. Cardiac vagal activity following three intensities of exercise in humans. Clin Physiol Funct Imaging. 2010;30:17-22.

12. Piras A, Cortesi M, Campa F, Perazzolo M, Gatta G. Recovery Time Profiling After Short-, Middle- and LongDistance Swimming Performance. J Strength Cond Res. 2019;33:1408-415.

13. Schaun GZ, Del Vecchio FB. High-Intensity Interval Exercises' Acute Impact on Heart Rate Variability: Comparison Between Whole-Body and Cycle Ergometer Protocols. J Strength Cond Res 2018;32:223-29.

14. Seiler S, Haugen O, Kuffel E. Autonomic recovery after exercise in trained athletes: intensity and duration effects. Med Sci Sports Exerc. 2007;39:1366-373.

15. Michael S, Jay O, Graham KS, Davis GM. Longer exercise duration delays post-exercise recovery of cardiac parasympathetic but not sympathetic indices. Eur J Appl Physiol. 2017;117:1897-906.

16. Araujo GS, Behm DG, Monteiro ER, Fiuza AGFM, Gomes TM, Vianna JM, Reis MS, Silva NJ. Order Effects of Resistance and Stretching Exercises on Heart Rate Variability and Blood Pressure in Healthy Adults. J Strength Cond Res. 2018;33(10):2684-693.

17. Figueiredo T, Willardson JM, Miranda H, Bentes CM, Reis VM, de Salles BF, Simao R Influence of Rest Interval Length Between Sets on Blood Pressure and Heart Rate Variability After a Strength Training Session Performed by Prehypertensive Men. J Strength Cond Res. 2016;30:1813-824.

18. Kingsley JD, Figueroa A. Acute and training effects of resistance exercise on heart rate variability. Clin Physiol Funct Imaging. 2014;36(3):179-87.

19. Figueiredo T, Rhea MR, Peterson M, Miranda H, Bentes CM, Reis VMR, Simão R. Influence of a number of sets on blood pressure and heart rate variability after a strength training session. J Strength Cond Res. 2015;29:1556-563.

20. Brown LE, Weir JP. ASEP procedures recommendation I: Accurate assessment of muscular strength and power. J Exerc Physiol. 2001;4:1-21.

21. Garber CE, Blissmer B, Deschenes MR, Franklin BA, Lamonte MJ, Lee IM, Nieman DC, Swain DP. American College of Sports Medicine position stand. Quantity and quality of exercise for developing and maintaining cardiorespiratory, musculoskeletal, and neuromotor fitness in apparently healthy adults: guidance for prescribing exercise. Med Sci Sports Exerc. 2011;43:1334-359.
22. Giles D, Draper N, Neil W. Validity of the Polar V800 heart rate monitor to measure RR intervals at rest. Eur J Appl Physiol. 2016;116:563-71.

23. Caminal P, Sola F, Gomis P, Guasch E, Perera A, Soriano N, Mont L. Validity of the Polar V800 monitor for measuring heart rate variability in mountain running route conditions. Eur J Appl Physiol. 2018;118:669-77.

24. Tarvainen MP, Niskanen JP, Lipponen JA, Ranta-Aho PO, Karjalainen PA. Kubios HRV--heart rate variability analysis software. Comput Methods Programs Biomed. 2014;113:210-20.

25. Cruz CJG, Porto LGG, Rolim PS, Pires DS, Garcia GL, Molina GE. Impact of heart rate on the reproducibility of heart rate variability analysis in the supine and standing positions in healthy men. Clinics. 2019;74:1-6.

26. Tulppo MP, Makikallio TH, Takala TE, Seppanen T, Huikuri HV. Quantitative beat-to-beat analysis of heart rate dynamics during exercise. Am J Physiol. 1996;271: 244-52.

27. Cruz CJG, Rolim PS, Pires DS, Mendes CMO, de Paula GM, Porto LGG, Garcia GL, Molina GE. Reliability of heart rate variability threshold and parasympathetic reactivation after a submaximal exercise test. Motriz: J. Phys. Ed. 2017;23:65-70.

28. Kaikkonen P, Hynynen E, Mann T, Rusko H, Nummela A. Can HRV be used to evaluate training load in constant load exercises? Eur J Appl Physiol. 2010;108:435-42.

29. Coote JH. Recovery of heart rate following intense dynamic exercise. Exp Physiol 2010;95:431-40.

30. Vesterinen V, Nummela A, Heikura I, Laine T, Hynynen E, Botella J, Häkkinen K. Individual Endurance Training Prescription with Heart Rate Variability. Med Sci Sports Exerc. 2016;48:1347-354.

31. Rinaldo MA, Jacinto JL, Pacagnelli FL, Shigaki L, Ribeiro AS, Balvedi MCW, Altimari LR, da Silva DK, de Andrade WB, da Silva RA, Aguiar AF. Effects of training volume on lower-body muscle strength in untrained young men: a contralateral control study. Motriz: J. Phys. Ed. 2018;24(3):1-7.

32. Martins-Costa HC, Diniz RCR, Lima FV, Machado SC, de Almeida RSV, de Andrade AGP, Chagas MH. Longer repetition duration increases muscle activation and blood lactate response in matched resistance training protocols. Motriz: J. Phys. Ed. 2016;22:35-41.

33. Sasaki K, Maruyama R. Consciously controlled breathing decreases the high-frequency component of heart rate variability by inhibiting cardiac parasympathetic nerve activity. Tohoku J Exp Med. 2014;233:155-63.

34. Agashe S, Petak S. Cardiac Autonomic Neuropathy in Diabetes Mellitus. Methodist Debakey Cardiovasc J. 2018;14:251-56.

35. Goldberger JJ, Arora R, Buckley U, Shivkumar K. Autonomic Nervous System Dysfunction: JACC Focus Seminar. J Am Coll Cardiol. 2019;73:1189-206.

36. Dahabreh IJ, Paulus JK. Association of episodic physical and sexual activity with triggering of acute cardiac events: systematic review and meta-analysis. JAMA. 2011;305:1225-233.

37. Marijon E, Uy-Evanado A, Reinier K, Teodorescu C, Narayanan K, Jouven X, Gunson K, Jui J, Chugh SS. Sudden cardiac arrest during sports activity in middle age. Circulation. 2015;131:1384-91. 


\section{Corresponding author}

Carlos Janssen Gomes da Cruz

Centro Universitário Euro Americano-UNIEURO - Group of Studies and Research in Cardiac Autonomic Function, Quadra Central. Conj B. Bloco C. Apto 104. Brasília, DF. 73010020, Brazil

Email: janssengomes@gmail.com

Editor: Katia de Angelis, UNIFESP, São Paulo, SP, Brasil
Manuscript received on February 20, 2020

Manuscript accepted on June 16, 2020

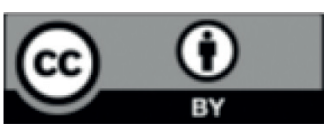

Motriz. The Journal of Physical Education. UNESP. Rio Claro, SP, Brazil - eISSN: 1980-6574 - under a license Creative Commons - Version 4.0 